\title{
Best Simultaneous Approximation to Totally Bounded Sequences in Banach Spaces *
}

\author{
Xianfa Luo \\ School of Mathematics and Computer Science, Jishou University \\ Jishou 416000, China \\ Email: luoxianfaseu@163.com \\ Chong Li \\ Department of Mathematics, Zhejiang University \\ Hangzhou 310027, China \\ Email: cli@zju.edu.cn \\ Genaro Lopez \\ Department of Mathematics Analysis, University of Sevilla \\ Sevilla, Spain \\ Email: glopez@us.es
}

\begin{abstract}
This paper is concerned with the problem of best weighted simultaneous approximations to totally bounded sequences in Banach spaces. Characterization results from convex sets in Banach spaces are established under the assumption that the Banach space is uniformly smooth.
\end{abstract}

Keywords: best simultaneous approximation; characterization; uniqueness; uniform smoothness.

\section{Introduction}

The problems of best simultaneous approximations to a set of functions has recently been a subject of intensive study, see for example $[1,2,3,4,5]$ in the case of finite many of functions and $[6,7,8,9]$ in the case of infinite, respectively. The case of finitely many is also a special case of the vector-valued approximation studied by Pinkus [10]. Here we are particularly interested in the kind of the best simultaneous approximation problems studied in $[1,2,4,8,9]$. The general setting of this kind problem is as follows. Let $1 \leq m \leq \infty$ and let $\mathbb{R}^{m}$ be a Banach space consisting of real $m$-tuple of vectors in the case when $m<\infty$ and some real sequences in the case when $m=\infty$ with the monotonic norm $\|\cdot\|_{A}$. Let $\left(\lambda_{v}\right)$ be a fixed element of $\mathbb{R}^{m}$ with each $\lambda_{v}>0$. Let $(X,\|\cdot\|)$ be a Banach space over the field $\mathbb{F}$, where $\mathbb{F}=\mathbb{R}$, the reals, or $\mathbb{F}=\mathbb{C}$, the complex

*The second author was supported in part by the National Natural Science Foundation of China (Grant No.10271025) and Program for New Century Excellent Talent in University; the third author was supported in part by projects BFM2000-0344 and FQM-127 of Spain. 
plane. Let $G$ be a fixed subset of $X$ and let $\hat{x}=\left(x_{v}\right)$ be a sequence of $X$ such that $\left(\lambda_{v}\left\|x_{v}\right\|\right) \in \mathbb{R}^{\infty}$. Then the problem concerned here is to finding an element $g_{0} \in G$ such that

$$
\left\|\left(\lambda_{v}\left\|x_{v}-g_{0}\right\|\right)\right\|_{A} \leq\left\|\left(\lambda_{v}\left\|x_{v}-g\right\|\right)\right\|_{A} \quad \text { for all } g \in G \text {. }
$$

Any element $g_{0}$ satisfying (1.1) is called a best simultaneous approximation to $\hat{x}$ from $G$. The set of all best simultaneous approximations to $\hat{x}$ from $G$ is denoted by $P_{G}(\hat{x})$. In the special case when $m=2$, this problem of approximating simultaneously continuous functions on a finite closed interval was first studied by Dunham in [1], where results on characterization and uniqueness of the best simultaneous approximation were obtained, while characterization and uniqueness results for a class of problems involving $L_{p}$ norms were given in [4]. A general treatment of a class of problems for the case when $m=2$, which includes these problems in [1, 4] as special cases, was given in [2]. Extensions to the case when $m=\infty$ have been considered in [8] for some special infinite sequences in a real Banach space, and in [9] for the general infinite sequences in a (real or complex) Banach space.

However, the study in $[8,9]$ for the problem of best simultaneous approximations to infinite sequences is based on the following key assumption:

$$
\lim _{v \rightarrow \infty}\left\|\left(0, \ldots, 0, \lambda_{v}, \lambda_{v+1}, \ldots\right)\right\|_{A}=0 .
$$

Thus one interesting question arises naturally: can the assumption (1.2) be dropped in the study of simultaneous approximations to infinite sequences? This problem seems very difficult for the general case. In fact, in the case when the assumption (1.2) is dropped, the method used in $[8,9]$ does not work. In the present paper, we shall always assume that $m=\infty$ and develop a completely different technique to investigate the problem of best simultaneous approximations to totally bounded sequences in Banach spaces without assumption (1.2). Under the assumption that $X$ is uniformly smooth, some characterization results similar to those in [2] for the best simultaneous approximation from convex sets in Banach space are obtained.

\section{Preliminaries}

Let $(X,\|\cdot\|)$ be a Banach space over the field $\mathbb{F}$, where $\mathbb{F}=\mathbb{R}$ or $\mathbb{C}$, and $\left(\mathbb{R}^{\infty},\|\cdot\|_{A}\right)$ a Banach space consisting of some sequences in $\mathbb{R}$. We use $\left(\mathbb{R}^{\infty}\right)^{*}$ and $X^{*}$ to denote the duals of $\mathbb{R}^{\infty}$ and $X$, respectively. The inner product between $\mathbb{R}^{\infty}$ and $\left(\mathbb{R}^{\infty}\right)^{*}$ is denoted by $\langle\cdot, \cdot\rangle_{A}$ while, for each pair $(x, f)$ with $x \in X$ and $f \in X^{*}$, $f(x)$ stands for the inner product of $x$ and $f$. The unit balls of $\left(\mathbb{R}^{\infty}\right)^{*}$ and $X^{*}$ are respectively denoted by $V$ and $W$. For a subset $A$ of $X$, let $\bar{A}$ stand for the closure of $A$ and $\operatorname{ext} A$ for the set of all extreme points of $A$. Recall that the set of all clusters of $A$ is called the derived set of $A$, which is denoted by $D(A)$.

Let $\mathbb{N}$ be the set of all positive integers. Recall that $\|\cdot\|_{A}$ is monotonic if, for any $\left(a_{v}\right) \in \mathbb{R}^{\infty}$ and any real sequence $\left(b_{v}\right)$, the fact that $\left|b_{v}\right| \leq\left|a_{v}\right|$ for each $v \in \mathbb{N}$ implies that $\left(b_{v}\right) \in \mathbb{R}^{\infty}$ and $\left\|\left(b_{v}\right)\right\|_{A} \leq\left\|\left(a_{v}\right)\right\|_{A}$. Let $\hat{\lambda}=\left(\lambda_{v}\right)$ be a fixed element of $\mathbb{R}^{\infty}$. Throughout the whole paper, we always assume that the norm $\|\cdot\|_{A}$ is monotonic and that $\lambda_{v}>0$ for each $v \in \mathbb{N}$. Furthermore, without loss of generality, we assume that $\|\hat{\lambda}\|_{A}=1$.

Let $I$ be a subset of $\mathbb{N}$. We use $\mathbf{e}_{I}=\left(e_{v}\right)$ to represent the element of $\mathbb{R}^{\infty}$ defined by $e_{v}=1$ if $v \in I$ and $e_{v}=0$ otherwise. In particular, we write, for each $i \in \mathbb{N}, \mathbf{e}_{i}$ for $\mathbf{e}_{I}$ if $I=\{i\}$. Thus, for an element 
$\hat{a}=\left(a_{v}\right) \in \mathbb{R}^{\infty}, \hat{a}_{I}$ stands for an element of $\mathbb{R}^{\infty}$ defined by $\hat{a}_{I}=\sum_{i \in I} a_{i} \mathbf{e}_{i}$. Let

$$
\mathcal{F}=\left\{\hat{x}=\left(x_{v}\right):\left(\lambda_{v}\left\|x_{v}\right\|\right) \in \mathbb{R}^{\infty}\right\}
$$

and let $\mathcal{F}$ be endowed with the norm $\|\cdot\|_{\mathcal{F}}$ defined by

$$
\|\hat{x}\|_{\mathcal{F}}=\left\|\left(\lambda_{v}\left\|x_{v}\right\|\right)\right\|_{A}, \quad \forall \hat{x}=\left(x_{v}\right) \in \mathcal{F} .
$$

Then $\left(\mathcal{F},\|\cdot\|_{\mathcal{F}}\right)$ is a Banach space. Note that $X$ can be embedded as a subset of $\mathcal{F}$ in a natural way that $\hat{x}=(x) \in \mathcal{F}$ for each $x \in X$. An element $\hat{x}=\left(x_{v}\right) \in \mathcal{F}$ is called a simple element of $\mathcal{F}$ if $\left\{x_{v}: v \in \mathbb{N}\right\}$ is a finite set. The set of all simple elements of $\mathcal{F}$ is denoted by $\mathcal{F}_{S}$. Recall that a finite class $\left\{I_{1}, \ldots, I_{m}\right\}$ of nonempty subsets of $\mathbb{N}$ is a partition of $\mathbb{N}$ if

$$
\bigcup_{i=1}^{m} I_{i}=\mathbb{N} \quad \text { and } \quad I_{i} \bigcap I_{j}=\emptyset, \quad \forall i \neq j .
$$

Then an element $\hat{x} \in \mathcal{F}_{S}$ if and only if there exist a finite subset $\left\{y_{1}, \ldots, y_{m}\right\}$ of $X$ and a partition $\left\{I_{1}, \ldots, I_{m}\right\}$ of $\mathbb{N}$ such that

$$
\hat{x}=\sum_{i=1}^{m} y_{i} \mathbf{e}_{I_{i}} .
$$

Clearly, if $\hat{x} \in \mathcal{F}_{S}$ is given by (2.1), then

$$
\|\hat{x}\|_{\mathcal{F}}=\left\|\sum_{i=1}^{m}\right\| y_{i}\left\|\hat{\lambda}_{I_{i}}\right\|_{A} .
$$

Let $\mathcal{F}_{T}$ denote the set of all elements $\hat{x}=\left(x_{v}\right)$ such that $\left\{x_{v}\right\}$ is totally bounded and let $\mathcal{F}_{T}$ endowed with the norm $\|\cdot\|_{\infty}$ defined by

$$
\|\hat{x}\|_{\infty}=\sup _{v \geq 1}\left\|x_{v}\right\|, \quad \forall \hat{x}=\left(x_{v}\right) \in \mathcal{F}_{T} .
$$

It is clear that

$$
\|\hat{x}\|_{\mathcal{F}} \leq\|\hat{x}\|_{\infty}, \quad \forall \hat{x}=\left(x_{v}\right) \in \mathcal{F}_{T} .
$$

Let $\overline{\mathcal{F}}_{S}^{T}$ denote the closure of $\mathcal{F}_{S}$ under the norm $\|\cdot\|_{\infty}$. Then the following relationships are clear.

Proposition 2.1. $X \subseteq \mathcal{F}_{S} \subseteq \overline{\mathcal{F}}_{S}^{T}=\mathcal{F}_{T} \subseteq \mathcal{F}$.

Let $m \in \mathbb{N}$ and let $\mathcal{I}=\left\{I_{i}: i=1, \ldots, m\right\}$ be a partition of $\mathbb{N}$. Set

$$
\mathcal{F}_{\mathcal{I}}^{m}=\left\{\hat{x} \in \mathcal{F}_{S}: \hat{x}=\sum_{i=1}^{m} y_{i} \mathbf{e}_{I_{i}},\left\{y_{1}, \ldots, y_{m}\right\} \subseteq X\right\} .
$$

Then $X \subseteq \mathcal{F}_{\mathcal{I}}^{m}$. Let $\Omega_{m}=V \times W^{m}$ and let $\Omega_{m}$ be endowed with the product topology, where $V$ and $W$ are respectively endowed with the weak* topology. Then $\Omega_{m}$ is a compact Hausdorff space. Let $C\left(\Omega_{m}\right)$ denote the Banach space of all real-valued continuous functions with the Chebyshev norm $\|\phi\|_{C}$ defined by

$$
\|\phi\|_{C}=\max _{\omega \in \Omega_{m}}|\phi(\omega)|, \quad \forall \phi \in C\left(\Omega_{m}\right) .
$$


For $\hat{x}=\sum_{i=1}^{m} y_{i} \mathbf{e}_{I_{i}} \in \mathcal{F}_{\mathcal{I}}^{m}$, define

$$
\phi_{\hat{x}}(\omega)=\sum_{i=1}^{m}\left\langle a^{*}, \hat{\lambda}_{I_{i}}\right\rangle_{A} \operatorname{Re} h_{i}\left(y_{i}\right), \quad \omega=\left(a^{*}, h_{1}, \ldots, h_{m}\right) \in \Omega_{m},
$$

where and through the whole paper, Re $b$ is read as $b$ in the case when $b$ is a real number. It is easy to see that $\phi_{\hat{x}} \in C\left(\Omega_{m}\right)$. Define a mapping $\Phi: \mathcal{F}_{\mathcal{I}}^{m} \rightarrow C\left(\Omega_{m}\right)$ by

$$
\Phi(\hat{x})=\phi_{\hat{x}}, \quad \forall \hat{x} \in \mathcal{F}_{\mathcal{I}}^{m}
$$

Then we have the following lemma.

Lemma 2.1. $\Phi$ is a linear isometry from $\mathcal{F}_{\mathcal{I}}^{m}$ to $\Phi\left(\mathcal{F}_{\mathcal{I}}^{m}\right) \subseteq C\left(\Omega_{m}\right)$

Proof. Note that the linearity of $\Phi$ is trivial. It suffices to show that the mapping is an isometry. To do this, let $\hat{x} \in \mathcal{F}_{\mathcal{I}}^{m}$. Since the norm $\|\cdot\|_{A}$ is monotonic, one has that

$$
\left\|\sum_{i=1}^{m} \hat{\lambda}_{I_{i}}\right\| y_{i}\|\|_{A}=\max \left\{\left\|\sum_{i=1}^{m} \hat{\lambda}_{I_{i}} \operatorname{Re} h_{i}\left(y_{i}\right)\right\|_{A}: h_{1}, \ldots, h_{m} \in W\right\} .
$$

Consequently, by $(2.2)$,

$$
\begin{aligned}
\|\hat{x}\|_{\mathcal{F}} & =\max \left\{\left\|\sum_{i=1}^{m} \hat{\lambda}_{I_{i}} \operatorname{Re}_{i}\left(y_{i}\right)\right\|_{A}: h_{1}, \ldots, h_{m} \in W\right\} \\
& =\max \left\{\left\langle a^{*}, \sum_{i=1}^{m} \hat{\lambda}_{I_{i}} \operatorname{Re}_{i}\left(y_{i}\right)\right\rangle_{A}:\left(a^{*}, h_{1}, \ldots, h_{m}\right) \in \Omega_{m}\right\} \\
& =\|\Phi(\hat{x})\|_{C} .
\end{aligned}
$$

Hence $\Phi$ is isometric. The proof is complete.

The following proposition, which is clearly a direct consequence of Lemma 2.1, converts equivalently the problem of the best simultaneous approximation to simple elements of $\mathcal{F}$ into that of the best Chebyshev approximation in $C\left(\Omega_{m}\right)$.

Proposition 2.2. Let $G$ be a nonempty subset of $X$. Then, for each $\hat{x} \in \mathcal{F}_{\mathcal{I}}^{m}$ and each $g_{0} \in G, g_{0}$ is a best simultaneous approximation to $\hat{x}$ from $G$ if and only if $\Phi\left(g_{0}\right)$ is a best Chebyshev approximation to $\Phi(\hat{x})$ from $\Phi(G)$.

\section{Characterizations of best simultaneous approximations}

We begin with the following notations. Let $y \in X$ and $\hat{x}=\left(x_{v}\right) \in \mathcal{F}$. Set

$$
W_{0}(y)=\{f \in W: f(y)=\|y\|\}
$$




$$
\begin{aligned}
& V_{0}(\hat{x})=\left\{a^{*} \in V:\left\langle a^{*},\left(\lambda_{v}\left\|x_{v}\right\|\right)\right\rangle_{A}=\|\hat{x}\|_{\mathcal{F}}\right\} \\
& N(\hat{x})=V_{0}(\hat{x}) \times \prod_{v=1}^{\infty} W_{0}\left(x_{v}\right), \\
& \widehat{N}(\hat{x})=\operatorname{ext} V_{0}(\hat{x}) \times \prod_{v=1}^{\infty} \operatorname{ext} W_{0}\left(x_{v}\right) .
\end{aligned}
$$

Let $m \in \mathbb{N}$ and let $\mathcal{I}=\left\{I_{i}: i=1, \ldots, m\right\}$ be a partition of $\mathbb{N}$. Let $\hat{x}=\sum_{i=1}^{m} y_{i} \mathbf{e}_{I_{i}} \in \mathcal{F}_{\mathcal{I}}^{m}$. We set

$$
N_{\mathcal{I}}^{m}(\hat{x})=\left\{\left(a^{*}, \hat{f}\right) \in N(\hat{x}): \begin{array}{l}
\hat{f}=\sum_{i=1}^{m} h_{i} \mathbf{e}_{I_{i}} \text { with } h_{i} \in W_{0}\left(y_{i}\right) \text { and } \\
\left\langle a^{*}, \hat{\lambda}_{I_{i}}\right\rangle_{A} \geq 0 \text { for each } i=1, \ldots, m
\end{array}\right\} .
$$

The first theorem of this section is concerned with the characterization of Kolmogorov type of the best simultaneous approximation to a simple element of $\mathcal{F}$ from a convex subset of $X$.

Theorem 3.1. Let $G$ be a convex subset of $X$. Let $\hat{x}=\sum_{i=1}^{m} y_{i} \mathbf{e}_{I_{i}} \in \mathcal{F}_{\mathcal{I}}^{m}$ and $g_{0} \in G$. Then the following statements are equivalent.

(i) $g_{0} \in P_{G}(\hat{x})$.

(ii) For each $g \in G$,

$$
\max \left\{\left\langle a^{*},\left(\operatorname{Re} \lambda_{v} f_{v}\left(g_{0}-g\right)\right)\right\rangle_{A}:\left(a^{*}, \hat{f}\right) \in N_{\mathcal{I}}^{m}\left(\hat{x}-g_{0}\right)\right\} \geq 0 .
$$

(iii) For each $g \in G$,

$$
\max \left\{\left\langle a^{*},\left(\operatorname{Re} \lambda_{v} f_{v}\left(g_{0}-g\right)\right)\right\rangle_{A}:\left(a^{*}, \hat{f}\right) \in N\left(\hat{x}-g_{0}\right)\right\} \geq 0 .
$$

Proof. (i) $\Longrightarrow\left(\right.$ ii). Suppose that $g_{0} \in P_{G}(\hat{x})$. Then, by Proposition $2.2, \Phi\left(g_{0}\right)$ is a best Chebyshev approximation to $\Phi(\hat{x})$ from $\Phi(G)$. Applying the well-known Kolmogorov characterization theorem for best Chebyshev approximations (cf. [11, Theorem 1]), we conclude that, for each $g \in G \backslash\left\{g_{0}\right\}$, there exists $\omega^{\prime}=\left(a^{\prime *}, h_{1}^{\prime}, \ldots, h_{m}^{\prime}\right) \in \Omega_{m}$ such that

$$
\phi_{\hat{x}}\left(\omega^{\prime}\right)-\phi_{g_{0}}\left(\omega^{\prime}\right)=\left\|\phi_{\hat{x}}-\phi_{g_{0}}\right\|_{C}
$$

and

$$
\phi_{g_{0}}\left(\omega^{\prime}\right)-\phi_{g}\left(\omega^{\prime}\right) \geq 0 .
$$

In view of (2.3), it follows from (3.2) and (3.3) that $\sum_{i=1}^{m}\left\langle a^{\prime *}, \hat{\lambda}_{I_{i}}\right\rangle_{A} \operatorname{Re} h_{i}^{\prime}\left(y_{i}-g_{0}\right)=\left\|\phi_{\hat{x}}-\phi_{g_{0}}\right\|_{C}$ and

$$
\sum_{i=1}^{m}\left\langle a^{\prime *}, \hat{\lambda}_{I_{i}}\right\rangle_{A} \operatorname{Re} h_{i}^{\prime}\left(g_{0}-g\right) \geq 0 .
$$

Set $s_{i}=\operatorname{sign}\left\langle a^{\prime *}, \hat{\lambda}_{I_{i}}\right\rangle_{A}$ for each $i=1, \ldots, m$. Then

$$
\begin{aligned}
\left\|\phi_{\hat{x}}-\phi_{g_{0}}\right\|_{C} & =\sum_{i=1}^{m}\left\langle a^{\prime *}, \hat{\lambda}_{I_{i}}\right\rangle_{A} \operatorname{Re} h_{i}^{\prime}\left(y_{i}-g_{0}\right) \\
& \leq \sum_{i=1}^{m}\left|\left\langle a^{\prime *}, \hat{\lambda}_{I_{i}}\right\rangle_{A}\right|\left|\operatorname{Re} h_{i}^{\prime}\left(y_{i}-g_{0}\right)\right| \\
& \leq \sum_{i=1}^{m}\left|\left\langle a^{\prime *}, \hat{\lambda}_{I_{i}}\right\rangle_{A}\right|\left\|y_{i}-g_{0}\right\| \\
& =\left\langle a^{\prime *}, \sum_{i=1}^{m} \hat{\lambda}_{I_{i}} s_{i}\left\|y_{i}-g_{0}\right\|\right\rangle_{A} \\
& \leq\left\|\sum_{i=1}^{m} \hat{\lambda}_{I_{i}} s_{i}\right\| y_{i}-g_{0}\|\|_{A} \\
& \leq\left\|\hat{x}-g_{0}\right\|_{\mathcal{F}},
\end{aligned}
$$


where the last inequality is because of $(2.2)$ and the monotonicity of the norm $\|\cdot\|_{A}$. Since $\left\|\phi_{\hat{x}}-\phi_{g_{0}}\right\|_{C}=$ $\left\|\hat{x}-g_{0}\right\|_{\mathcal{F}}$ by Lemma 2.1, the inequalities in (3.5) are equalities. Consequently, one has that

$$
\sum_{i=1}^{m}\left|\left\langle a^{\prime *}, \hat{\lambda}_{I_{i}}\right\rangle_{A}\right|\left\|y_{i}-g_{0}\right\|=\left\|\hat{x}-g_{0}\right\|_{\mathcal{F}}
$$

and, for each $i=1, \ldots, m$ with $s_{i} \neq 0$,

$$
s_{i} \operatorname{Re}_{i}^{\prime}\left(y_{i}-g_{0}\right) \geq 0, \quad\left|\operatorname{Re}_{i}^{\prime}\left(y_{i}-g_{0}\right)\right|=\left|h_{i}^{\prime}\left(y_{i}-g_{0}\right)\right|=\left\|y_{i}-g_{0}\right\| .
$$

Below we will construct $\left(a^{*}, \hat{f}\right) \in N_{\mathcal{I}}^{m}\left(\hat{x}-g_{0}\right)$ such that $\left\langle a^{*},\left(\operatorname{Re} \lambda_{v} f_{v}\left(g_{0}-g\right)\right)\right\rangle_{A} \geq 0$. Granting this, (3.1) follows. To do this, define the linear functional $a^{*}$ on $\mathbb{R}^{\infty}$ by

$$
\left\langle a^{*}, \hat{b}\right\rangle_{A}=\left\langle a^{\prime *}, \sum_{i=1}^{m} s_{i} \hat{b}_{I_{i}}\right\rangle_{A}, \quad \forall \hat{b}=\left(b_{v}\right) \in \mathbb{R}^{\infty}
$$

Note that, by the monotonicity of the norm $\|\cdot\|_{A},\left|\left\langle a^{*}, \hat{b}\right\rangle_{A}\right| \leq\left\|\sum_{i=1}^{m} s_{i} \hat{b}_{I_{i}}\right\|_{A} \leq\|\hat{b}\|_{A}$; hence we have $a^{*} \in V$. In addition, we have that

$$
\left\langle a^{*}, \hat{\lambda}_{I_{i}}\right\rangle_{A}=\left\langle a^{* *}, \hat{\lambda}_{I_{i}}\right\rangle_{A} s_{i}=\left|\left\langle a^{\prime *}, \hat{\lambda}_{I_{i}}\right\rangle_{A}\right| \geq 0, \quad \forall i=1, \ldots, m
$$

Let $\hat{f}=\sum_{i=1}^{m} h_{i} \mathbf{e}_{I_{i}}$, where $h_{i} \in W_{0}\left(y_{i}-g_{0}\right)$ if $s_{i}=0$ and $h_{i}=s_{i} h_{i}^{\prime}$ if $s_{i} \neq 0$. Then $\left(a^{*}, \hat{f}\right)$ is desired. In fact, since, by (3.6) and (3.8),

$$
\left\langle a^{*},\left(\lambda_{v}\left\|x_{v}-g_{0}\right\|\right)\right\rangle_{A}=\sum_{i=1}^{m}\left\langle a^{*}, \hat{\lambda}_{I_{i}}\right\rangle_{A}\left\|y_{i}-g_{0}\right\|=\sum_{i=1}^{m}\left|\left\langle a^{\prime *}, \hat{\lambda}_{I_{i}}\right\rangle_{A}\right|\left\|y_{i}-g_{0}\right\|=\left\|\hat{x}-g_{0}\right\|_{\mathcal{F}},
$$

one has that $a^{*} \in V_{0}\left(\hat{x}-g_{0}\right)$. In view of the definition of $h_{i},(3.7)$ implies that $h_{i} \in W_{0}\left(y_{i}-g_{0}\right)$ for each $i=1, \ldots, m$; hence, $\left(a^{*}, \hat{f}\right) \in N_{\mathcal{I}}^{m}\left(\hat{x}-g_{0}\right)$ thanks to (3.8). On the other hand, the definitions of $h_{1}, h_{2}, \cdots, h_{m}$ together with (3.8) imply that

$$
\sum_{i=1}^{m}\left\langle a^{*}, \hat{\lambda}_{I_{i}}\right\rangle_{A} \operatorname{Re}_{i}\left(g_{0}-g\right)=\sum_{i=1}^{m}\left\langle a^{\prime *}, \hat{\lambda}_{I_{i}}\right\rangle_{A} \operatorname{Re}_{i}^{\prime}\left(g_{0}-g\right) .
$$

Thus (3.1) holds by (3.4) and (i) $\Longrightarrow$ (ii) is proved.

(ii) $\Longrightarrow$ (iii). It is trivial.

(iii) $\Longrightarrow$ (i). Suppose that (iii) holds and let $g \in G \backslash\left\{g_{0}\right\}$ be arbitrary. Then there is $\left(a^{*}, f_{1}, f_{2}, \ldots\right) \in$ $N\left(\hat{x}-g_{0}\right)$ such that $\left\langle a^{*},\left(\operatorname{Re} \lambda_{v} f_{v}\left(g_{0}-g\right)\right)\right\rangle_{A} \geq 0$. Hence,

$$
\begin{aligned}
\left\|\hat{x}-g_{0}\right\|_{\mathcal{F}} & =\left\langle a^{*},\left(\operatorname{Re} \lambda_{v} f_{v}\left(x_{v}-g_{0}\right)\right)\right\rangle_{A} \\
& =\left\langle a^{*},\left(\operatorname{Re} \lambda_{v} f_{v}\left(x_{v}-g\right)\right)\right\rangle_{A}+\left\langle a^{*},\left(\operatorname{Re} \lambda_{v} f_{v}\left(g-g_{0}\right)\right)\right\rangle_{A} \\
& \leq\|\hat{x}-g\|_{\mathcal{F}}
\end{aligned}
$$

This means that $g_{0} \in P_{G}(\hat{x})$ and (iii) $\Longrightarrow(\mathbf{i})$ is proved. The proof is complete.

For the next theorem of this section, we recall that a Banach space $(X,\|\cdot\|)$ is uniformly smooth if, for any $\epsilon>0$ there exists $\delta>0$ such that $\|x+y\|+\|x-y\|-2<\|y\| \epsilon$ holds for any $x, y \in X$ with $\|x\|=1$ and 
$0<\|y\|<\delta$. Note that a Banach space which is uniformly smooth is reflexive. Let $\sigma: X \rightarrow 2^{W}$ denote the supporting mapping defined by $\sigma(x)=\{f \in W: f(x)=\|x\|\}$ for each $x \in X$. Then the following characterization result about the uniform smoothness of a Banach space is known in [12, Theorem 1, P.36].

Proposition 3.1. A Banach space $X$ is uniformly smooth if and only if the supporting mapping $\sigma$ is single-valued and norm-norm uniformly continuous on the unit sphere of $X$.

Let $g, \bar{g} \in G$ and $\hat{x} \in \mathcal{F}$. Set

$$
I(\bar{g})=\left\{v \in \mathbb{N}: x_{v}=\bar{g}\right\} .
$$

Suppose that $X$ is uniformly smooth and define a mapping $F_{\hat{x}-\bar{g}}: W \mapsto \prod_{v=1}^{\infty} W$ by

$$
F_{\hat{x}-\bar{g}}(w)=\hat{f}^{w}:=\sum_{v \in \mathbb{N} \backslash I(\bar{g})} \sigma\left(x_{v}-\bar{g}\right) \mathbf{e}_{v}+w \mathbf{e}_{I(\bar{g})} \quad \text { for each } w \in W .
$$

We write for convenience

$$
\begin{gathered}
K(\hat{x} ; \bar{g}, g)=\left\{\left(a^{*}, \hat{f}\right)=\left(a^{*}, F_{\hat{x}-\bar{g}}(w)\right): a^{*} \in V_{0}(\hat{x}-\bar{g}), w \in W_{0}(\bar{g}-g)\right\}, \\
M(\hat{x} ; \bar{g})=\left\{\left(a^{*}, \hat{f}\right)=\left(a^{*}, F_{\hat{x}-\bar{g}}(w)\right): a^{*} \in V_{0}(\hat{x}-\bar{g}), w \in W\right\}, \\
\widehat{K}(\hat{x} ; \bar{g}, g)=\left\{\left(a^{*}, \hat{f}\right)=\left(a^{*}, F_{\hat{x}-\bar{g}}(w)\right): a^{*} \in \operatorname{ext} V_{0}(\hat{x}-\bar{g}), w \in \operatorname{ext} W_{0}(\bar{g}-g)\right\}
\end{gathered}
$$

and

$$
\widehat{M}(\hat{x} ; \bar{g})=\left\{\left(a^{*}, \hat{f}\right)=\left(a^{*}, F_{\hat{x}-\bar{g}}(w)\right): a^{*} \in \operatorname{ext} V_{0}(\hat{x}-\bar{g}), w \in \operatorname{ext} W\right\} .
$$

Then

$$
N(\hat{x}-\bar{g}) \supseteq M(\hat{x} ; \bar{g}) \supseteq K(\hat{x} ; \bar{g}, g) \supseteq \widehat{K}(\hat{x} ; \bar{g}, g)
$$

and

$$
\widehat{N}(\hat{x}-\bar{g}) \supseteq \widehat{M}(\hat{x} ; \bar{g}) \supseteq \widehat{K}(\hat{x} ; \bar{g}, g) .
$$

Now we are ready to give the main theorem of this section. Recall that $D\left(\left\{x_{v}\right\}\right)$ denotes the derived set of $\left\{x_{v}\right\}$ and that $\left[g_{0}, g\right]$ the segment with endpoints $g_{0}$ and $g$. Consider the following conditions:

$$
\left\langle a^{*},\left(\operatorname{Re} \lambda_{v} f_{v}\left(x_{v}-g_{0}\right)\right)\right\rangle_{A}=\left\|\hat{x}-g_{0}\right\|_{\mathcal{F}}
$$

and

$$
\left\langle a^{*},\left(\operatorname{Re} \lambda_{v} f_{v}\left(g_{0}-g\right)\right)\right\rangle_{A} \geq 0 .
$$

Theorem 3.2. Let $G$ be a convex subset of $X$ and let $\hat{x}=\left(x_{v}\right) \in \mathcal{F}_{T}$ be such that $P_{G}(\hat{x}) \cap D\left(\left\{x_{v}\right\}\right)=\emptyset$. Suppose that $X$ is uniformly smooth. Then the following statements are equivalent.

(i) $g_{0} \in P_{G}(\hat{x})$.

(ii) For each $g \in G \backslash\left\{g_{0}\right\}$, there exist $\bar{g} \in P_{G}(\hat{x}) \cap\left[g_{0}, g\right]$ and $\left(a^{*}, \hat{f}\right) \in \widehat{K}(\hat{x} ; \bar{g}, g)$ such that $(3.12)$ and (3.13) hold. 
(ii*) For each $g \in G \backslash\left\{g_{0}\right\}$, there exist $\bar{g} \in P_{G}(\hat{x}) \cap\left[g_{0}, g\right]$ and $\left(a^{*}, \hat{f}\right) \in K(\hat{x} ; \bar{g}, g)$ such that $(3.12)$ and (3.13) hold.

(iii) For each $g \in G \backslash\left\{g_{0}\right\}$, there exist $\bar{g} \in P_{G}(\hat{x}) \cap\left[g_{0}, g\right]$ and $\left(a^{*}, \hat{f}\right) \in \widehat{M}(\hat{x} ; \bar{g})$ such that (3.12) and (3.13) hold.

(iii*) For each $g \in G \backslash\left\{g_{0}\right\}$, there exist $\bar{g} \in P_{G}(\hat{x}) \cap\left[g_{0}, g\right]$ and $\left(a^{*}, \hat{f}\right) \in M(\hat{x} ; \bar{g})$ such that (3.12) and (3.13) hold.

(iv) For each $g \in G \backslash\left\{g_{0}\right\}$, there exist $\bar{g} \in P_{G}(\hat{x}) \cap\left[g_{0}, g\right]$ and $\left(a^{*}, \hat{f}\right) \in \widehat{N}(\hat{x}-\bar{g})$ such that (3.12) and (3.13) hold.

(iv*) For each $g \in G \backslash\left\{g_{0}\right\}$, there exist $\bar{g} \in P_{G}(\hat{x}) \cap\left[g_{0}, g\right]$ and $\left(a^{*}, \hat{f}\right) \in N(\hat{x}-\bar{g})$ such that $(3.12)$ and (3.13) hold.

(v) For each $g \in G \backslash\left\{g_{0}\right\}$, there exists $\left(a^{*}, \hat{f}\right) \in \operatorname{ext} V \times \prod_{v=1}^{\infty} \operatorname{ext} W$ such that (3.12) and (3.13) hold.

$\left(\mathbf{v}^{*}\right)$ For each $g \in G \backslash\left\{g_{0}\right\}$, there exists $\left(a^{*}, \hat{f}\right) \in V \times \prod_{v=1}^{\infty} W$ such that (3.12) and (3.13) hold.

Proof. Clearly, the following implications hold by (3.10) and (3.11):

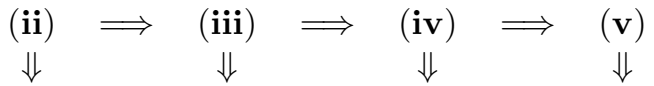

$$
\begin{aligned}
& \left(\mathrm{ii}^{*}\right) \Longrightarrow\left(\mathrm{iii}^{*}\right) \Longrightarrow\left(\mathbf{i v}^{*}\right) \Longrightarrow\left(\mathbf{v}^{*}\right)
\end{aligned}
$$

Thus, it suffices to verify the implications $(\mathbf{i}) \Longrightarrow(\mathbf{i i} *) \Longrightarrow(\mathbf{i i})$ and $\left(\mathbf{v}^{*}\right) \Longrightarrow(\mathbf{i})$.

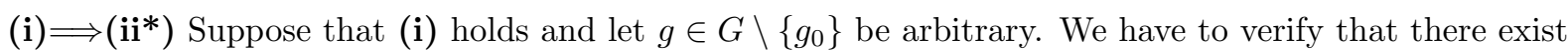
$\bar{g} \in P_{G}(\hat{x}) \cap\left[g_{0}, g\right]$ and $\left.\left(a^{*}, \hat{f}\right) \in K(\hat{x} ; \bar{g}, g)\right)$ such that (3.12) and (3.13) hold. For this purpose, note that, by Proposition 2.1 , for each $n$, there exists $\hat{x}^{n} \in \mathcal{F}_{S}$ such that $\left\|\hat{x}^{n}-\hat{x}\right\|_{\infty}<\frac{1}{n}$, or equivalently,

$$
\left\|x_{v}^{n}-x_{v}\right\|<\frac{1}{n}, \quad v=1,2, \ldots
$$

Let $g_{n}$ be a best approximation to $\hat{x}^{n}$ from $\left[g_{0}, g\right]$. Then $\left\{g_{n}\right\}$ is bounded and hence, without loss of generality, we may assume that $\left\{g_{n}\right\}$ converges to, say, $\bar{g}_{0}$. It is easy to see that $\bar{g}_{0} \in P_{G}(\hat{x}) \cap\left[g_{0}, g\right]$. Below we divide the proof into two cases: (a) $\bar{g}_{0} \neq g_{0}$ and (b) $\bar{g}_{0}=g_{0}$.

(a) $\bar{g}_{0} \neq g_{0}$. Set $\bar{g}=\frac{1}{2}\left(g_{0}+\bar{g}_{0}\right)$. Take $a^{*} \in V_{0}(\hat{x}-\bar{g})$ and $f \in W_{0}(\bar{g}-g)$. Define

$$
f_{v}= \begin{cases}\sigma\left(x_{v}-\bar{g}\right), & v \in \mathbb{N} \backslash I(\bar{g}), \\ f, & v \in I(\bar{g}) .\end{cases}
$$

Then $\left(a^{*}, \hat{f}\right) \in K(\hat{x} ; \bar{g}, g)$. Furthermore,

$$
\left\langle a^{*},\left(\operatorname{Re} \lambda_{v} f_{v}\left(\frac{1}{2}\left(\left(x_{v}-g_{0}\right)+\left(x_{v}-\bar{g}_{0}\right)\right)\right)\right)\right\rangle_{A}=\|\hat{x}-\bar{g}\|_{\mathcal{F}}
$$

This implies that

$$
\left\|\hat{x}-g_{0}\right\|_{\mathcal{F}}=\left\langle a^{*},\left(\operatorname{Re} \lambda_{v} f_{v}\left(x_{v}-g_{0}\right)\right)\right\rangle_{A}=\left\langle a^{*},\left(\operatorname{Re} \lambda_{v} f_{v}\left(x_{v}-\bar{g}_{0}\right)\right)\right\rangle_{A}=\left\|\hat{x}-\bar{g}_{0}\right\|_{\mathcal{F}}
$$

because $\|\hat{x}-\bar{g}\|_{\mathcal{F}}=\left\|\hat{x}-g_{0}\right\|_{\mathcal{F}}=\left\|\hat{x}-\bar{g}_{0}\right\|_{\mathcal{F}}$. Hence, by $(3.16),\left\langle a^{*},\left(\operatorname{Re} \lambda_{v} f_{v}\left(\bar{g}_{0}-g_{0}\right)\right)\right\rangle_{A}=0$, which implies that $\left\langle a^{*},\left(\operatorname{Re} \lambda_{v} f_{v}\left(g_{0}-g\right)\right)\right\rangle_{A}=0$. Hence (3.13) holds while (3.12) follows from (3.16).

(b) $\bar{g}_{0}=g_{0}$. Take $\bar{g}=g_{0}$. Let $n \in \mathbb{N}$ and assume that $\hat{x}^{n}=\sum_{i=1}^{k_{n}} y_{i}^{n} \mathbf{e}_{I_{i}^{n}}$, where $\left\{I_{i}^{n}: i=1, \ldots, k_{n}\right\}$ is a partition of $\mathbb{N}$. Recall that $I(\bar{g})=\left\{v: x_{v}=\bar{g}\right\}$. Furthermore, set

$$
I_{i 1}^{n}=I_{i}^{n} \cap I(\bar{g}) \quad \text { and } \quad I_{i 2}^{n}=I_{i}^{n} \backslash I(\bar{g}), \quad \forall i=1,2, \ldots, k_{n} .
$$


Then $\left\{I_{i k}^{n}: k=1,2 ; i=1, \ldots, k_{n}\right\}$ is a partition of $\mathbb{N}$ and $\hat{x}^{n}$ can be rewritten as

$$
\hat{x}^{n}=\sum_{i=1}^{k_{n}}\left(y_{i}^{n} \mathbf{e}_{I_{i 1}^{n}}+y_{i}^{n} \mathbf{e}_{I_{i 2}^{n}}\right) .
$$

Let $m_{n}=2 k_{n}$ and $\mathcal{I}_{n}=\left\{I_{i k}^{n}: k=1,2 ; i=1, \ldots, k_{n}\right\}$. Then $\hat{x}^{n} \in \mathcal{F}_{\mathcal{I}_{n}}^{m_{n}}$. Noting that $g_{n} \in P_{\left[g_{0}, g\right]}\left(\hat{x}^{n}\right)$, we can apply Theorem 3.1 to get that there exists $\left(a_{n}^{*}, \hat{f}^{n}\right) \in N_{\mathcal{I}_{n}}^{m_{n}}\left(\hat{x}^{n}-g_{n}\right)$ such that

$$
\left\langle a_{n}^{*},\left(\operatorname{Re} \lambda_{v} f_{v}^{n}\left(g_{n}-g\right)\right)\right\rangle_{A} \geq 0 .
$$

Assume that

$$
\hat{f}^{n}=\sum_{i=1}^{k_{n}}\left(h_{i 1}^{n} \mathbf{e}_{I_{i 1}^{n}}+h_{i 2}^{n} \mathbf{e}_{I_{i 2}^{n}}\right)
$$

where

$$
\left(a_{n}^{*},\left(h_{11}^{n}, h_{12}^{n}\right), \ldots,\left(h_{k_{n} 1}^{n}, h_{k_{n} 2}^{n}\right)\right) \in V_{0}\left(\hat{x}^{n}-g_{n}\right) \times \prod_{i=1}^{k_{n}}\left(W_{0}\left(y_{i}^{n}-g_{n}\right)\right)^{2}
$$

Then

$$
\left\langle a_{n}^{*}, \sum_{i=1}^{k_{n}} \hat{\lambda}_{I_{i 1}^{n}} \operatorname{Re}_{i 1}^{n}\left(g_{n}-g\right)\right\rangle_{A}+\left\langle a_{n}^{*}, \sum_{i=1}^{k_{n}} \hat{\lambda}_{I_{i 2}^{n}} \operatorname{Re}_{i 2}^{n}\left(g_{n}-g\right)\right\rangle_{A} \geq 0
$$

and

$$
\left\langle a_{n}^{*}, \hat{\lambda}_{I_{i k}^{n}}\right\rangle_{A} \geq 0, \quad k=1,2, \quad i=1, \ldots, k_{n} .
$$

Note that (3.17) implies that

$$
\left\langle a_{n}^{*},\left(\lambda_{v}\left\|x_{v}^{n}-g_{n}\right\|\right)\right\rangle_{A}=\left\|\hat{x}^{n}-g_{n}\right\|_{\mathcal{F}}
$$

and

$$
h_{i k}^{n}\left(y_{i}^{n}-g_{n}\right)=\left\|y_{i}^{n}-g_{n}\right\|, \quad k=1,2, \quad i=1, \ldots, k_{n} .
$$

Now, take $f \in W_{0}(\bar{g}-g)$ and let $\hat{f}=\left(f_{v}\right)$ where $\left\{f_{v}\right\}$ are defined by (3.15). Let $\hat{a}=\left(a_{v}\right), \hat{b}=\left(b_{v}\right)$ and $\hat{c}=\left(c_{v}\right)$ be defined respectively by $a_{v}=\lambda_{v}\left\|x_{v}-\bar{g}\right\|$ for each $v \in \mathbb{N}$,

$$
b_{v}= \begin{cases}0, & v \in \mathbb{N} \backslash I(\bar{g}), \\ \operatorname{Re} \lambda_{v} f_{v}\left(g_{0}-g\right), & v \in I(\bar{g})\end{cases}
$$

and

$$
c_{v}= \begin{cases}\operatorname{Re} \lambda_{v} f_{v}\left(g_{0}-g\right), & v \in \mathbb{N} \backslash I(\bar{g}), \\ 0, & v \in I(\bar{g})\end{cases}
$$

Noting that $\left\{a_{n}^{*}\right\} \subset V$, without loss of generality, we may assume that there exists $a^{*} \in V$ such that

$$
\lim _{n \rightarrow \infty}\left\langle a_{n}^{*}, \hat{d}\right\rangle_{A}=\left\langle a^{*}, \hat{d}\right\rangle_{A}, \quad \forall \hat{d} \in \operatorname{span}\{\hat{a}, \hat{b}, \hat{c}\}
$$

By (3.20), one has that

$$
\left\langle a^{*},\left(\lambda_{v}\left\|x_{v}-\bar{g}\right\|\right)\right\rangle_{A}-\left\|\hat{x}^{n}-g_{n}\right\|_{\mathcal{F}}=\left\langle a^{*}-a_{n}^{*}, \hat{a}\right\rangle_{A}+\left\langle a_{n}^{*},\left(\lambda_{v}\left(\left\|x_{v}-\bar{g}\right\|-\left\|x_{v}^{n}-g_{n}\right\|\right)\right\rangle_{A} .\right.
$$

Note that $\lim _{n \rightarrow \infty}\left\langle a_{n}^{*}, \hat{a}\right\rangle_{A} \rightarrow\left\langle a^{*}, \hat{a}\right\rangle_{A}$ by $(3.22)$ and

$$
\left|\left\langle a_{n}^{*},\left(\lambda_{v}\left(\left\|x_{v}-\bar{g}\right\|-\left\|x_{v}^{n}-g_{n}\right\|\right)\right)\right\rangle_{A}\right| \leq \frac{1}{n}+\left\|\bar{g}-g_{n}\right\| \rightarrow 0
$$


by (3.14). Note also that $g_{n} \rightarrow \bar{g}_{0}=\bar{g}$ strongly. Then taking limits on both sides of the equality above gives that $\left\langle a^{*},\left(\lambda_{v}\left\|x_{v}-\bar{g}\right\|\right)\right\rangle_{A}=\|\hat{x}-\bar{g}\|_{\mathcal{F}}$. This implies that $a^{*} \in V_{0}(\hat{x}-\bar{g})$. Hence $\left(a^{*}, \hat{f}\right) \in K(\hat{x} ; \bar{g}, g)$ and $(3.12)$ holds since $\bar{g}=g_{0}$. Thus, it remains to show that (3.13) holds. To do this, note that (3.13) is equivalent that

$$
\left\langle a^{*}, \hat{b}\right\rangle_{A}+\left\langle a^{*}, \hat{c}\right\rangle_{A} \geq 0
$$

By the definition of $f_{v}$ in $(3.15)$, one has that $\operatorname{Re} \lambda_{v} f_{v}(\bar{g}-g)=\lambda_{v}\|\bar{g}-g\|$ when $v \in I(\bar{g})$. This means that

$$
\hat{b}=\|\bar{g}-g\| \hat{\lambda}_{I(\bar{g})}=\sum_{i=1}^{k_{n}} \hat{\lambda}_{I_{i 1}^{n}}\|\bar{g}-g\| .
$$

Set

$$
\hat{b}^{n}=\sum_{i=1}^{k_{n}} \hat{\lambda}_{I_{i 1}^{n}} \operatorname{Re}_{i 1}^{n}(\bar{g}-g) \quad \text { and } \quad \hat{c}^{n}=\sum_{i=1}^{k_{n}} \hat{\lambda}_{I_{i 2}^{n}} \operatorname{Re}_{i 2}^{n}(\bar{g}-g) .
$$

Then (3.18) implies that

$$
\limsup _{n \rightarrow \infty}\left\langle a_{n}^{*}, \hat{b}^{n}\right\rangle_{A}+\limsup _{n \rightarrow \infty}\left\langle a_{n}^{*}, \hat{c}^{n}\right\rangle_{A} \geq 0
$$

because $g_{n} \rightarrow \bar{g}$ strongly. Since by (3.19) and (3.24)

$$
\left\langle a_{n}^{*}, \hat{b}^{n}\right\rangle_{A}=\sum_{i=1}^{k_{n}}\left\langle a_{n}^{*}, \hat{\lambda}_{I_{i 1}^{n}}\right\rangle_{A} \operatorname{Re}_{i 1}^{n}(\bar{g}-g) \leq \sum_{i=1}^{k_{n}}\left\langle a_{n}^{*}, \hat{\lambda}_{I_{i 1}^{n}}\right\rangle_{A}\|\bar{g}-g\|=\left\langle a_{n}^{*}, \hat{b}\right\rangle_{A},
$$

it follows that $\limsup _{n \rightarrow \infty}\left\langle a_{n}^{*}, \hat{b}^{n}\right\rangle_{A} \leq\left\langle a^{*}, \hat{b}\right\rangle_{A}$. Therefore, by (3.25), to complete the proof of (3.23), it suffices to verify that

$$
\lim _{n \rightarrow \infty}\left\langle a_{n}^{*}, \hat{c}^{n}\right\rangle_{A}=\left\langle a^{*}, \hat{c}\right\rangle_{A}
$$

To show (3.26), for each $n \in \mathbb{N}$, define $\hat{f}^{n}=\left(f_{v}^{n}\right)$ by

$$
\hat{f}^{n}=\sum_{i=1}^{k_{n}} h_{i 2}^{n} \mathbf{e}_{I_{i 2}^{n}}
$$

Then

$$
\hat{c}^{n}=\left(\operatorname{Re} \lambda_{v} f_{v}^{n}(\bar{g}-g)\right) .
$$

Let $\varepsilon>0$ be arbitrary. Since $X$ is uniformly smooth, one has from Proposition 3.1 that the supporting mapping $\sigma: X \rightarrow W$ is norm-norm uniformly continuous on the unit sphere $S(X)$ of $X$. This implies that there exists $\delta>0$ such that

$$
\|\sigma(x)-\sigma(y)\|<\varepsilon, \quad \forall x, y \in S(X) \text { with }\|x-y\|<\delta .
$$

Note that $\delta_{0}:=\inf _{v \in \mathbb{N} \backslash I(\bar{g})}\left\|x_{v}-\bar{g}\right\|>0$ as $P_{G}(\hat{x}) \cap D\left(\left\{x_{v}\right\}\right)=\emptyset$ and $\bar{g} \in P_{G}(\hat{x})$. Note also that $\left\|g_{n}-\bar{g}\right\| \rightarrow 0$ and $\sup _{v \in \mathbb{N}}\left\|x_{v}^{n}-x_{v}\right\| \leq \frac{1}{n}$ by (3.14). Without loss of generality, we may assume that $x_{v}^{n} \neq g_{n}$ for each $n \in \mathbb{N}$ and $v \in \mathbb{N} \backslash I(\bar{g}))$. Let $v \in \mathbb{N} \backslash I(\bar{g}))$. Consider the sequence $\left\{u_{v}^{n}\right\} \subset S(X)$ defined by

$$
u_{v}^{n}=\frac{x_{v}^{n}-g_{n}}{\left\|x_{v}^{n}-g_{n}\right\|} \quad \text { for each } n \in \mathbb{N} .
$$


Then

$$
f_{v}^{n}=\sigma\left(u_{v}^{n}\right), \quad \forall n \in \mathbb{N}
$$

thanks to (3.21) and (3.27). Write $u_{v}=\frac{x_{v}-\bar{g}}{\left\|x_{v}-\bar{g}\right\|}$. Then, $f_{v}=\sigma\left(u_{v}\right)$ by the definition of $\hat{f}$. Let $N \in \mathbb{N}$ be such that

$$
\frac{1}{n}+\left\|g_{n}-\bar{g}\right\|<\delta_{0} \quad \text { and } \quad 2 \frac{\frac{1}{n}+\left\|g_{n}-\bar{g}\right\|}{\delta_{0}-\frac{1}{n}-\left\|\bar{g}-g_{n}\right\|}<\delta, \quad \forall n \geq N .
$$

Then, estimating the norm of $u_{v}^{n}-u_{v}$, we have that, for each $n \geq N$,

$$
\begin{aligned}
\left\|u_{v}^{n}-u_{v}\right\| & =\left\|\frac{x_{v}^{n}-g_{n}}{\left\|x_{v}^{n}-g_{n}\right\|}-\frac{x_{v}-\bar{g}}{\left\|x_{v}-\bar{g}\right\|}\right\| \\
& =\left\|\frac{\left\|x_{v}-\bar{g}\right\|\left(x_{v}^{n}-g_{n}-\left(x_{v}-\bar{g}\right)\right)-\left(\left\|x_{v}^{n}-g_{n}\right\|-\left\|x_{v}-\bar{g}\right\|\right)\left(x_{v}-\bar{g}\right)}{\left\|x_{v}^{n}-g_{n}\right\|\left\|x_{v}-\bar{g}\right\|}\right\| \\
& \leq \frac{\left\|x_{v}^{n}-g_{n}-\left(x_{v}-\bar{g}\right)\right\|+\left\|x_{v}^{n}-g_{n}\right\|-\left\|x_{v}-\bar{g}\right\|}{\left\|x_{v}-\bar{g}+\bar{g}-g_{n}+x_{v}^{n}-x_{v}\right\|} \\
& \leq 2 \frac{\left\|x_{v}^{n}-x_{v}\right\|+\left\|g_{n}-\bar{g}\right\|}{\left\|x_{v}-\bar{g}\right\|-\left\|\bar{g}-g_{n}\right\|-\left\|x_{v}^{n}-x_{v}\right\|} \\
& \leq 2 \frac{\frac{1}{n}+\left\|g_{n}-\bar{g}\right\|}{\delta_{0}-\frac{1}{n}-\left\|\bar{g}-g_{n}\right\|}<\delta .
\end{aligned}
$$

This together with (3.28) implies that $\left\|f_{v}^{n}-f_{v}\right\|<\varepsilon$ for each $n>N$ and so

$$
\left\|f_{v}^{n}-f_{v}\right\|<\varepsilon \text { for all } n>N, v \in \mathbb{N} \backslash I(\bar{g}) .
$$

Therefore,

$$
\left|c_{v}^{n}-c_{v}\right| \leq \lambda_{v}\|\bar{g}-g\| \varepsilon
$$

holds for all $n>N$ and $v \in \mathbb{N} \backslash I(\bar{g})$. Since, for each $n \in \mathbb{N}$ and $v \in I(\bar{g}), c_{v}^{n}=c_{v}=0$, (3.29) holds for all $n>N$ and $v \in \mathbb{N}$. Consequently, one has that

$$
\left|\left\langle a_{n}^{*}, \hat{c}^{n}\right\rangle_{A}-\left\langle a^{*}, \hat{c}\right)\right\rangle_{A}|\leq|\left\langle a_{n}^{*}-a^{*}, \hat{c}\right\rangle_{A} \mid+\|\bar{g}-g\| \varepsilon, \quad \forall n>N
$$

Because $\left|\left\langle a_{n}^{*}-a^{*}, \hat{c}\right\rangle_{A}\right| \rightarrow 0$ by (3.22) and $\varepsilon>0$ is arbitrary, (3.26) is seen to hold. Hence the proof of $(\mathrm{i}) \Longrightarrow\left(\mathrm{ii}^{*}\right)$ is complete.

(ii*) $\Longrightarrow\left(\right.$ ii). Suppose that (ii*) holds and $g \in G \backslash\left\{g_{0}\right\}$. Then there exist $\bar{g} \in P_{G}(\hat{x}) \cap\left[g_{0}, g\right]$ and $\left(a^{*}, \hat{f}\right) \in K(\hat{x} ; \bar{g}, g)$ such that (3.12) and (3.13) hold. According to the definition of $K(\hat{x} ; \bar{g}, g)$, we have that $f_{v}=\sigma\left(x_{v}-\bar{g}\right)$ for each $v \in \mathbb{N} \backslash I(\bar{g})$ and there exists $f \in W_{0}(\bar{g}-g)$ such that $f_{v}=f$ for all $v \in I(\bar{g})$. Define $\hat{d}=\left(d_{v}\right)$ by

$$
d_{v}= \begin{cases}0, & v \in I(\bar{g}) \\ \operatorname{Re} \lambda_{v} f_{v}\left(g_{0}-g\right), & v \in \mathbb{N} \backslash I(\bar{g})\end{cases}
$$

Then

$$
\left\langle a^{*},\left(\operatorname{Re} \lambda_{v} f_{v}\left(g_{0}-g\right)\right)\right\rangle_{A}=\left\langle a^{*}, \hat{\lambda}_{I(\bar{g})}\right\rangle_{A} \operatorname{Re} f\left(g_{0}-g\right)+\left\langle a^{*}, \hat{d}\right\rangle_{A},
$$

which together with (3.13) implies that

$$
\left\langle a^{*}, \hat{\lambda}_{I(\bar{g})}\right\rangle_{A} \operatorname{Re} f\left(g_{0}-g\right)+\left\langle a^{*}, \hat{d}\right\rangle_{A} \geq 0 .
$$


Consequently,

$$
\alpha:=\max \left\{\left\langle a^{*}, \hat{\lambda}_{I(\bar{g})}\right\rangle_{A} \operatorname{Re} f\left(g_{0}-g\right)+\left\langle a^{*}, \hat{d}\right\rangle_{A}:\left(a^{*}, f\right) \in V_{0}(\hat{x}-\bar{g}) \times W_{0}(\bar{g}-g)\right\} \geq 0 .
$$

Note that

$$
\alpha=\max _{a^{*} \in V_{0}(\hat{x}-\bar{g})} \max _{f \in W_{0}(\bar{g}-g)}\left[\left\langle a^{*}, \hat{\lambda}_{I(\bar{g})}\right\rangle_{A} \operatorname{Re} f\left(g_{0}-g\right)+\left\langle a^{*}, \hat{d}\right\rangle_{A}\right],
$$

and note also that the function

$$
a^{*} \mapsto \max _{f \in W_{0}(\bar{g}-g)}\left[\left\langle a^{*}, \hat{\lambda}_{I(\bar{g})}\right\rangle_{A} \operatorname{Re} f\left(g_{0}-g\right)+\left\langle a^{*}, \hat{d}\right\rangle_{A}\right]
$$

is a convex continuous function on the compact convex set $V_{0}(\hat{x}-\bar{g})$. The Krein-Milman theorem $(c f$. [13, Theorem, P.74]) is applicable to concluding that there exists $a_{0}^{*} \in \operatorname{ext} V_{0}(\hat{x}-\bar{g})$ such that

$$
\alpha=\max _{f \in W_{0}(\bar{g}-g)}\left[\left\langle a_{0}^{*}, \hat{\lambda}_{I(\bar{g})}\right\rangle_{A} \operatorname{Re} f\left(g_{0}-g\right)+\left\langle a_{0}^{*}, \hat{d}\right\rangle_{A}\right]
$$

Similarly, there is $f^{0} \in \operatorname{ext} W_{0}(\bar{g}-g)$ such that

$$
\alpha=\left\langle a_{0}^{*}, \hat{\lambda}_{I(\bar{g})}\right\rangle_{A} \operatorname{Re} f^{0}\left(g_{0}-g\right)+\left\langle a_{0}^{*}, \hat{d}\right\rangle_{A} .
$$

Consequently, $\left(a_{0}^{*}, \hat{f}^{0}\right) \in \widehat{K}(\hat{x} ; \bar{g}, g)$ and (3.12) and $(3.13)$ hold with $\left(a_{0}^{*}, \hat{f}^{0}\right)$ in place of $\left(a^{*}, \hat{f}\right)$ thanks to (3.30) and (3.31), which completes the proof of (ii*) $\Longrightarrow(\mathbf{i i})$.

$\left(\mathbf{v}^{*}\right) \Longrightarrow(\mathbf{i})$ Suppose that $\left(\mathbf{v}^{*}\right)$ holds. Then, for each $g \in G \backslash\left\{g_{0}\right\}$, there exists $\left(a^{*}, \hat{f}\right) \in V \times \prod_{v=1}^{\infty} W$ such that (3.12) and (3.13) hold. Consequently,

$$
\begin{aligned}
\left\|\hat{x}-g_{0}\right\|_{\mathcal{F}} & =\left\langle a^{*},\left(\operatorname{Re} \lambda_{v} f_{v}\left(x_{v}-g\right)\right)\right\rangle_{A}+\left\langle a^{*},\left(\operatorname{Re} \lambda_{v} f_{v}\left(g-g_{0}\right)\right)\right\rangle_{A} \\
& \leq\left\langle a^{*},\left(\operatorname{Re} \lambda_{v} f_{v}\left(x_{v}-g\right)\right)\right\rangle_{A} \\
& \leq\|\hat{x}-g\|_{\mathcal{F}}
\end{aligned}
$$

This means that $g_{0} \in P_{G}(\hat{x})$ because $g \in G \backslash\left\{g_{0}\right\}$ is arbitrary and hence $\left(\mathbf{v}^{*}\right) \Longrightarrow(\mathbf{i})$ is proved.

Recall that the norm $\|\cdot\|_{A}$ in $\mathbb{R}^{\infty}$ is strictly monotonic if, $\|\cdot\|_{A}$ is monotonic and, for any $\hat{a}=\left(a_{v}\right), \hat{b}=$ $\left(b_{v}\right) \in \mathbb{R}^{\infty}$ with $0 \leq a_{v} \leq b_{v}$ for each $v \in \mathbb{N}$, the condition $\|\hat{a}\|_{A}=\|\hat{b}\|_{A}$ implies that $\hat{a}=\hat{b}$.

Corollary 3.1. Let $G$ be a convex subset of $X$ and let $\hat{x}=\left(x_{v}\right) \in \mathcal{F}_{T}$ be such that $P_{G}(\hat{x}) \cap D\left(\left\{x_{v}\right\}\right)=\emptyset$. Suppose that $X$ is uniformly smooth and the norm $\|\cdot\|_{A}$ in $\mathbb{R}^{\infty}$ is strictly monotonic. Then the following statements are equivalent.

(i) $g_{0} \in P_{G}(\hat{x})$.

(ii) $\max \left\{\left\langle a^{*},\left(\operatorname{Re} \lambda_{v} f_{v}\left(g_{0}-g\right)\right)\right\rangle_{A}:\left(a^{*}, \hat{f}\right) \in \widehat{K}\left(\hat{x} ; g_{0}, g\right)\right\} \geq 0$ for each $g \in G$.

$\left(\tilde{\mathbf{i i}}^{*}\right) \max \left\{\left\langle a^{*},\left(\operatorname{Re} \lambda_{v} f_{v}\left(g_{0}-g\right)\right)\right\rangle_{A}:\left(a^{*}, \hat{f}\right) \in K\left(\hat{x} ; g_{0}, g\right)\right\} \geq 0$ for each $g \in G$.

(iii) $\max \left\{\left\langle a^{*},\left(\operatorname{Re} \lambda_{v} f_{v}\left(g_{0}-g\right)\right)\right\rangle_{A}:\left(a^{*}, \hat{f}\right) \in \widehat{M}\left(\hat{x} ; g_{0}\right)\right\} \geq 0$ for each $g \in G$.

$\left(\tilde{\text { iii }}^{*}\right) \max \left\{\left\langle a^{*},\left(\operatorname{Re} \lambda_{v} f_{v}\left(g_{0}-g\right)\right)\right\rangle_{A}:\left(a^{*}, \hat{f}\right) \in M\left(\hat{x} ; g_{0}\right)\right\} \geq 0$ for each $g \in G$.

(iv) $\max \left\{\left\langle a^{*},\left(\operatorname{Re} \lambda_{v} f_{v}\left(g_{0}-g\right)\right)\right\rangle_{A}:\left(a^{*}, \hat{f}\right) \in \widehat{N}\left(\hat{x}-g_{0}\right)\right\} \geq 0$ for each $g \in G$.

$\left(\tilde{\mathbf{i v}}^{*}\right) \max \left\{\left\langle a^{*},\left(\operatorname{Re} \lambda_{v} f_{v}\left(g_{0}-g\right)\right)\right\rangle_{A}:\left(a^{*}, \hat{f}\right) \in N\left(\hat{x}-g_{0}\right)\right\} \geq 0$ for each $g \in G$. 
Proof. Clearly, by (3.10) and (3.11),

$$
\begin{array}{ccccc}
(\tilde{\mathbf{i i}}) & \Longrightarrow & (\tilde{\mathbf{i i i}}) & \Longrightarrow & (\tilde{\mathbf{i v}}) \\
\Downarrow & & & \Downarrow \\
\left(\tilde{\tilde{i i}}^{*}\right) & \Longrightarrow & \left(\tilde{\mathbf{i i i}}^{*}\right) & \Longrightarrow & \left(\tilde{\mathbf{i v}}^{*}\right)
\end{array}
$$

Clearly $\left(\tilde{\mathbf{i v}}^{*}\right)$ implies $\left(\mathbf{i v}^{*}\right)$ of Theorem 3.2. Hence $\left(\tilde{\mathbf{i v}}^{*}\right) \Longrightarrow(\mathbf{i})$ follows from Theorem 3.2. Thus, to complete the proof, it remains to show that $(\mathbf{i}) \Longrightarrow(\tilde{\mathbf{i i}})$. To do this, let $g_{0} \in P_{G}(\hat{x})$ and $g \in G \backslash\left\{g_{0}\right\}$ be arbitrary. Then, by Theorem 3.2 (ii), there exist $\bar{g} \in P_{G}(\hat{x}) \cap\left[g_{0}, g\right]$ and $\left(a^{*}, \hat{f}^{\prime}\right) \in \widehat{K}(\hat{x} ; \bar{g}, g)$ such that $(3.12)$ and (3.13) hold with $\left(a^{\prime *}, \hat{f}^{\prime}\right)$ in place of $\left(a^{*}, \hat{f}\right)$. This clearly implies that

$$
\max \left\{\left\langle a^{*},\left(\operatorname{Re} \lambda_{v} f_{v}\left(g_{0}-g\right)\right)\right\rangle_{A}:\left(a^{*}, \hat{f}\right) \in \widehat{K}\left(\hat{x} ; g_{0}, g\right)\right\} \geq 0
$$

in the case when $\bar{g}=g_{0}$. Hence, we may assume that $\bar{g} \neq g_{0}$. Since $\|\cdot\|_{A}$ is monotonic, one has that

$$
\left\|\left(\operatorname{Re} \lambda_{v} f_{v}^{\prime}\left(x_{v}-g_{0}\right)\right)\right\|_{A}=\left\|\left(\left|\operatorname{Re} \lambda_{v} f_{v}^{\prime}\left(x_{v}-g_{0}\right)\right|\right)\right\|_{A} \leq\left\|\left(\lambda_{v}\left\|x_{v}-g_{0}\right\|\right)\right\|_{A}=\left\|\hat{x}-g_{0}\right\|_{\mathcal{F}}
$$

By (3.12), we have that

$$
\left\|\hat{x}-g_{0}\right\|_{\mathcal{F}}=\left\langle a^{\prime *},\left(\operatorname{Re} \lambda_{v} f_{v}^{\prime}\left(x_{v}-g_{0}\right)\right)\right\rangle_{A} \leq\left\|\left(\operatorname{Re} \lambda_{v} f_{v}^{\prime}\left(x_{v}-g_{0}\right)\right)\right\|_{A} .
$$

Combining (3.33) and (3.34) yields that

$$
\left\|\left(\left|\operatorname{Re} \lambda_{v} f_{v}^{\prime}\left(x_{v}-g_{0}\right)\right|\right)\right\|_{A}=\left\|\left(\lambda_{v}\left\|x_{v}-g_{0}\right\|\right)\right\|_{A}
$$

which together with the strict monotonicity of the norm $\|\cdot\|_{A}$ implies that

$$
\left|\operatorname{Re} f_{v}^{\prime}\left(x_{v}-g_{0}\right)\right|=\left\|x_{v}-g_{0}\right\|, \quad \forall v \in \mathbb{N} .
$$

Recall that $I\left(g_{0}\right)=\left\{v: x_{v}=g_{0}\right\}$. Since $\bar{g} \neq g_{0}$, we have that $I(\bar{g}) \cap I\left(g_{0}\right)=\emptyset$. It follows that

$$
f_{v}^{\prime}=\sigma\left(x_{v}-\bar{g}\right)=\sigma\left(g_{0}-\bar{g}\right)=\sigma\left(g_{0}-g\right), \quad \forall v \in I\left(g_{0}\right)
$$

because $\bar{g} \in\left(g_{0}, g\right]$. Define $\hat{f}=\left(f_{v}\right)$ by $f_{v}=s_{v} f_{v}^{\prime}$ for each $v \in \mathbb{N}$, where

$$
s_{v}= \begin{cases}1, & v \in I\left(g_{0}\right), \\ \operatorname{signRe} f_{v}^{\prime}\left(x_{v}-g_{0}\right), & v \in \mathbb{N} \backslash I\left(g_{0}\right) .\end{cases}
$$

Then, by (3.35) and (3.36),

$$
f_{v}= \begin{cases}\sigma\left(g_{0}-g\right) & \text { for each } v \in I\left(g_{0}\right) \\ \sigma\left(x_{v}-g_{0}\right) & \text { for each } v \in \mathbb{N} \backslash I\left(g_{0}\right)\end{cases}
$$

That is, $\hat{f}=F_{\hat{x}-g_{0}}\left(\sigma\left(g_{0}-g\right)\right)$. Now define the linear functional $a^{*}$ on $\mathbb{R}^{\infty}$ by

$$
\left\langle a^{*}, \hat{b}\right\rangle_{A}=\left\langle a^{\prime *},\left(s_{v} b_{v}\right)\right\rangle_{A}, \quad \forall \hat{b}=\left(b_{v}\right) \in \mathbb{R}^{\infty} .
$$

Then $a^{*} \in \operatorname{ext} V$. In fact, otherwise, there exist $a_{1}^{*}, a_{2}^{*} \in V$ such that $a^{*}=\frac{1}{2}\left(a_{1}^{*}+a_{2}^{*}\right)$. Define

$$
\left\langle a_{i}^{\prime *}, \hat{b}\right\rangle_{A}=\left\langle a_{i}^{*},\left(s_{v} b_{v}\right)\right\rangle_{A}, \quad \forall \hat{b}=\left(b_{v}\right) \in \mathbb{R}^{\infty} \text { and } i=1,2 .
$$


Then $a_{1}^{\prime *}, a_{2}^{\prime *} \in V$ and $a^{\prime *}=\frac{1}{2}\left(a_{1}^{\prime *}+a_{2}^{\prime *}\right)$, which contradicts that $a^{\prime *} \in \operatorname{ext} V$. Furthermore, by (3.34), we have that

$$
\left\langle a^{*},\left(\lambda_{v}\left\|x_{v}-g_{0}\right\|\right)\right\rangle_{A}=\left\langle a^{\prime *},\left(\operatorname{Re} \lambda_{v} f_{v}^{\prime}\left(x_{v}-g_{0}\right)\right)\right\rangle_{A}=\left\|\hat{x}-g_{0}\right\|_{\mathcal{F}} .
$$

It follows that $a^{*} \in V_{0}\left(\hat{x}-g_{0}\right)$. Hence $\left(a^{*}, \hat{f}\right) \in \widehat{K}\left(\hat{x} ; g_{0}, g\right)$ because $\hat{f}=F_{\hat{x}-g_{0}}\left(\sigma\left(g_{0}-g\right)\right)$. On the other hand, since, by (3.12) and (3.39),

$$
\begin{aligned}
\left\langle a^{\prime *},\left(\operatorname{Re} \lambda_{v} f_{v}^{\prime}\left(g_{0}-\bar{g}\right)\right)\right\rangle_{A} & =\left\langle a^{\prime *},\left(\operatorname{Re} \lambda_{v} f_{v}^{\prime}\left(x_{v}-\bar{g}\right)\right)\right\rangle_{A}-\left\langle a^{\prime *},\left(\operatorname{Re} \lambda_{v} f_{v}^{\prime}\left(x_{v}-g_{0}\right)\right)\right\rangle_{A} \\
& =\|\hat{x}-\bar{g}\|_{\mathcal{F}}-\left\|\hat{x}-g_{0}\right\|_{\mathcal{F}}=0,
\end{aligned}
$$

we get that

$$
\left\langle a^{*},\left(\operatorname{Re} \lambda_{v} f_{v}\left(g_{0}-\bar{g}\right)\right)\right\rangle_{A}=\left\langle a^{\prime *},\left(\operatorname{Re} \lambda_{v} f_{v}^{\prime}\left(g_{0}-\bar{g}\right)\right)\right\rangle_{A}=0 .
$$

This means that

$$
\left\langle a^{*},\left(\operatorname{Re} \lambda_{v} f_{v}\left(g_{0}-g\right)\right)\right\rangle_{A}=0
$$

because $g_{0}-g=t\left(g_{0}-\bar{g}\right)$ for some $t \in \mathbb{R}$. Therefore, (3.32) holds in the case when $\bar{g} \neq g_{0}$ and the proof of (i) $\Longrightarrow(\tilde{\mathbf{i i}})$ is complete.

Recall that $X$ is said to be strictly convex if $\|(x+y) / 2\|<1$ for any $x, y \in X$ with $\|x\| \leq 1$ and $\|y\| \leq 1$. Thus when $\mathbb{R}^{\infty}$ is strictly convex, Corollary 3.1 can be improved to the following Corollary 3.2.

Corollary 3.2. Let $G$ be a convex subset of $X, g_{0} \in G$ and let $\hat{x}=\left(x_{v}\right) \in \mathcal{F}_{T}$ be such that $g_{0} \notin D\left(\left\{x_{v}\right\}\right)$. Suppose that $X$ is uniformly smooth and $\mathbb{R}^{\infty}$ is strictly convex. Then the statements in Corollary 3.1 are equivalent.

Proof. Note that strict convexity of $\mathbb{R}^{\infty}$ implies the strict monotonicity of the norm $\|\cdot\|_{A}$. In fact, otherwise, there exist two distinct elements $\hat{a}=\left(a_{v}\right), \hat{b}=\left(b_{v}\right) \in \mathbb{R}^{\infty}$ with $0 \leq a_{v} \leq b_{v}$ for each $v \in \mathbb{N}$ such that $\|\hat{a}\|_{A}=\|\hat{b}\|_{A}$. Since

$$
0 \leq a_{v} \leq \frac{1}{2}\left(a_{v}+b_{v}\right) \leq b_{v}, \quad \forall v \in \mathbb{N},
$$

it follows from the monotonicity of the norm $\|\cdot\|_{A}$ that

$$
\|\hat{a}\|_{A} \leq\left\|\frac{1}{2}(\hat{a}+\hat{b})\right\|_{A} \leq\|\hat{b}\|_{A}=\|\hat{a}\|_{A},
$$

which contradicts that $\mathbb{R}^{\infty}$ is strictly convex. In order to apply Corollary 3.1 , we have to verify that $P_{G}(\hat{x}) \cap D\left(\left\{x_{v}\right\}\right)=\emptyset$. To do this, let $\bar{g} \in P_{G}(\hat{x})$. Without loss of generality, we may assume that $\bar{g} \neq g_{0}$. Since $G$ is convex, we have that

$$
\begin{aligned}
\left\|\hat{x}-g_{0}\right\|_{\mathcal{F}} & =\left\|\hat{x}-\frac{g_{0}+\bar{g}}{2}\right\|_{\mathcal{F}} \\
& \leq\left\|\frac{1}{2}\left(\lambda_{v}\left\|x_{v}-g_{0}\right\|\right)+\frac{1}{2}\left(\lambda_{v}\left\|x_{v}-\bar{g}\right\|\right)\right\|_{A} \\
& \leq \frac{1}{2}\left\|\left(\lambda_{v}\left\|x_{v}-g_{0}\right\|\right)\right\|_{A}+\frac{1}{2}\left\|\left(\lambda_{v}\left\|x_{v}-\bar{g}\right\|\right)\right\|_{A} \\
& =\left\|\hat{x}-g_{0}\right\|_{\mathcal{F}} .
\end{aligned}
$$


It follows from the strict convexity of $\mathbb{R}^{\infty}$ that

$$
\left\|x_{v}-g_{0}\right\|=\left\|x_{v}-\bar{g}\right\|, \quad \forall v \in \mathbb{N} .
$$

Therefore, $\bar{g} \notin D\left(\left\{x_{v}\right\}\right)$ thanks to the fact that $g_{0} \notin D\left(\left\{x_{v}\right\}\right)$; hence $P_{G}(\hat{x}) \cap D\left(\left\{x_{v}\right\}\right)=\emptyset$ is proved. Consequently, Corollary 3.1 is applicable to concluding that the statements in Corollary 3.1 are equivalent.

\section{Concluding remark}

We have establish some characterizations for best simultaneous approximation, which are completely in view of the elements from the unit balls of $\left(\mathbb{R}^{\infty}\right)^{*}$ and $X^{*}$. There is another approach to studying this problem, which is considered as a best approximation in the new normed linear space $\left(\mathcal{F},\|\cdot\|_{\mathcal{F}}\right)$ defined in Section 2. In fact, write $\hat{G}=\{(g): g \in G\} \subseteq \mathcal{F} . g_{0} \in G$ is a best simultaneous approximation to $\hat{x}=\left(x_{v}\right)$ from $G$ if and only if it is a best (single) approximation to $\hat{x}$ for $\hat{G}$ in $\left(\mathcal{F},\|\cdot\|_{\mathcal{F}}\right)$. Thus applying the characterization results for the convex best approximation problem in $\left(\mathcal{F},\|\cdot\|_{\mathcal{F}}\right)$, one can easily get the following trivial result:

Theorem 4.1. $g_{0} \in G$ is a best simultaneous approximation to $\hat{x}=\left(x_{v}\right)$ from $G$ if and only if

$$
\left.\max \left\{\left\langle f^{*},\left(g_{0}-g\right)\right)\right\rangle_{\mathcal{F}}: f^{*} \in M\left(\hat{x}-g_{0}\right)\right\} \geq 0, \quad \forall g \in G
$$

where

$$
M\left(\hat{x}-g_{0}\right)=\left\{f^{*} \in \operatorname{ext} \mathcal{M}^{*}:\left\langle f^{*},\left(x_{v}-g_{0}\right)\right\rangle_{\mathcal{F}}=\left\|\hat{x}-g_{0}\right\|_{\mathcal{F}}\right\}
$$

and $\mathcal{M}^{*}$ is the unit ball of $\mathcal{F}^{*}$.

However, since it is impossible in general to express the elements in $M\left(\hat{x}-g_{0}\right)$ with the elements from $V$ and $W$, one can not deduce, from Theorem 4.1, the characterizations presented in previous section. Clearly, Theorem 4.1 is less convenient in applications.

\section{References}

[1] C. B. Dunham, Simultaneous Chebyshev approximation of functions on an interval, Proc. Amer. Math. Soc. 18 (1967) 472-477.

[2] C. Li and G. A. Watson, On a class of best simultaneous approximation, Comput. Math. Appl. 31 (1996) 45-53.

[3] C. Li and G. A. Watson, On best simultaneous approximation, J. Approx. Theory 91 (1997) 332-348.

[4] J. Shi and R. Huotari, Simultaeous approximation from convex sets, Computers Math. Applic. 30 (1995) 197-206. 
[5] G. A. Watson, A characterization of best simultaneous approximations, J. Approx. Theory 75 (1993) 175-182.

[6] C. Li and G. A. Watson, Best simultaneous approximation of an infinite set of functions, Comput. Math. Appl. 37 (1999) 1-9.

[7] C. Li and G. A. Watson, On nonlinear simultaneous Chebyshev approximation problems, J. Math. Anal. Appl. 288 (2003) 167-181.

[8] X. F. Luo, J. S. He and C. Li, On best simultaneous approximation from suns to an infinite sequence in Banach space, Acta Math. Sinica 45 (2002) 287-294 (in Chinese).

[9] X. F. Luo, C. Li and G. Lopez, Nonlinear weighted best simultaneous approximation in Banach spaces, submitted to J. Math. Anal. Appl.

[10] A. Pinkus, Uniqueness in vector-valued approximation, J. Approx. Theory 73 (1993) 17-92.

[11] J. H. Freilich and H. W. Mclaughlin, Approximation of bounded sets, J. Approx. Theory 34 (1982) 146-158.

[12] J. Diestel, Geometry of Banach Spaces-Selected Topics, Lecture Notes in Mathematics 485, SpringerVerlag, 1975.

[13] R. B. Holmes, Geometrical Functional and Applications, Springer-Verlag, 1975.

[14] X. F. Luo, C. Li and G. Lopez, Uniqueness results of best simultaneous approximation from $R S$-sets in Banach spaces, Preprint. 